\title{
PSRSalsa and the bi-drifting pulsar B1839-04
}

\author{
Patrick Weltevrede \\ Jodrell Bank Centre for Astrophysics, The University of Manchester \\ Alan Turing Building, Manchester, M13 9PL, United Kingdom \\ email: Patrick.Weltevrede@manchester.ac.uk
}

\begin{abstract}
PSRSalsa is a versatile open-source pulsar data-analysis project designed to obtain a comprehensive picture of the radio properties of your pulsar of choice. Here its usefulness is demonstrated through the analysis of the radio pulsar B1839-04, thereby revealing the extremely rare phenomenon of "bi-drifting" where the drift direction of subpulses is systematically different in different pulse profile components.
\end{abstract}

Keywords. methods: data analysis, pulsars: individual (B1839-04), pulsars: general

\section{Bi-drifting in PSR B1839-04}

PSR B1839-04 is a 1.84 second pulsar and its pulse-to-pulse radio variability is fascinating (Weltevrede 2016). From $1380 \mathrm{MHz}$ Westerbork Synthesis Radio Telescope observations it is apparent that the pulsar periodically brightens because of subpulse modulation (see left panel of Fig. 1). The later part of the sequence is highly periodic, while earlier the characteristics of the pulses were radically different (known as a mode change). PSR B1839-04 shows a very peculiar type of subpulse modulation known as bi-drifting where different profile components show subpulse drift in opposite directions. Although subpulse modulation itself is common, bi-drifting is extremely rare and is only observed for one other pulsar: PSR J0815+0939 (Champion et al. 2005).

Bi-drifting in PSR B1839-04 is not immediately evident from visual inspection of the pulse sequence, hence a more sophisticated analysis is conducted using the opensource pulsar data-analysis project PSRSalsa (Weltevrede 2016). Here we demonstrate the existence of bi-drifting using spectral analysis. The top right panels of Fig. 1 show the two-dimensional fluctuation spectrum for the two profile components respectively. Both spectra show a feature at $\sim 0.08 P / P_{3}$, implying a repetition rate $P_{3}$ in the drifting subpulse pattern of 12 pulses. The feature is offset to the left in the first plot, but to the right in the second plot (especially the second harmonic shown between the dashed lines). This implies bi-drifting as can more directly be visualised by " $P_{3}$-folding" the data with the 12 pulse periodicity (while correcting for its variability), resulting in the average of the repeating pattern as shown in the bottom right panel of Fig. 1 . The $P_{3}$-fold result confirms that the trailing component is drifting slightly in the opposite direction.

\section{Implications}

Understanding the physics of these results is difficult because the modulation of the two components is strictly phase-locked: observations years apart show the same relative alignment of the modulation patterns in the two components, despite the mode changes regularly destroying these patterns. This implies that a single physical origin is responsible for the modulation pattern in both components. This rules out interpretations based 

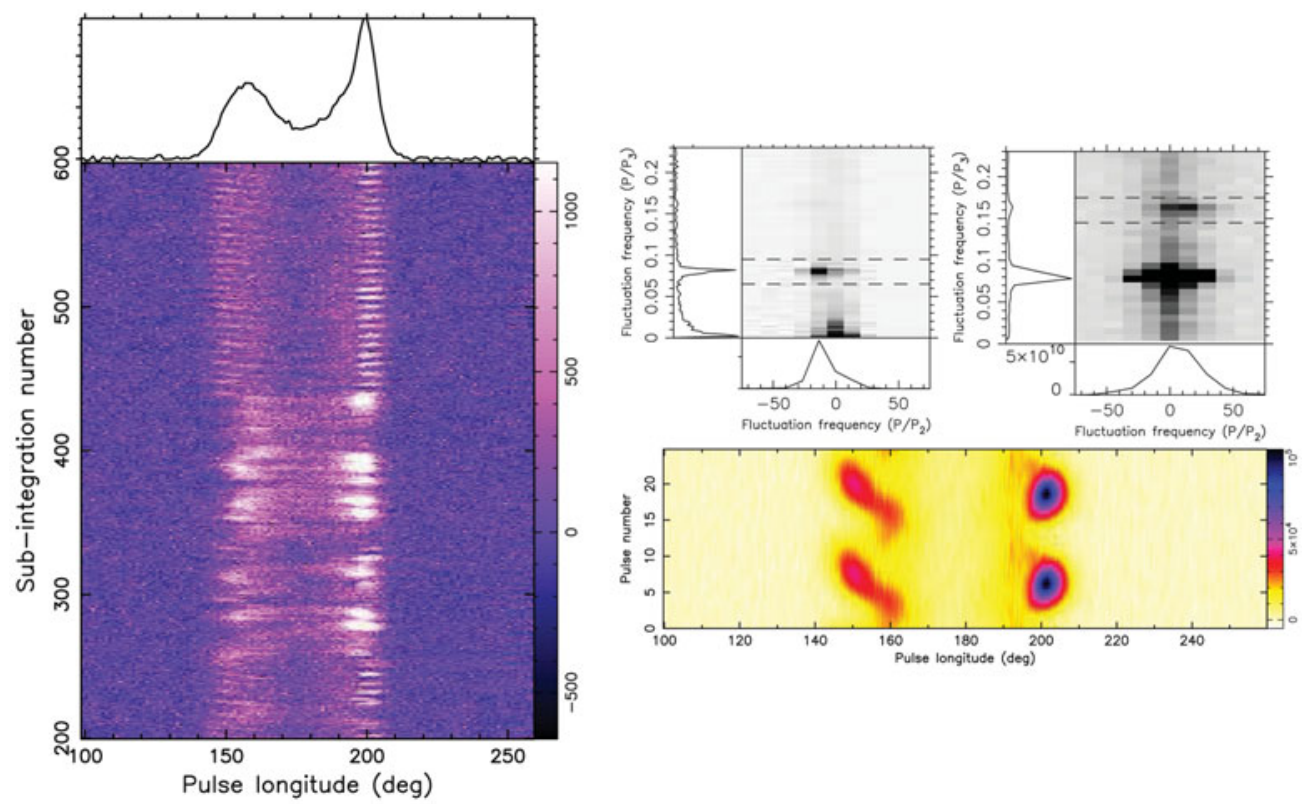

Figure 1. Left: A pulse-sequence of PSR B1839-04 with every pair of pulses being summed. Top right: Two two-dimensional fluctuation spectra. The left/right spectrum is that of the leading/trailing profile component. Bottom right: A $P_{3}$ fold of the data.

on oppositely rotating nested subbeam systems (with or without invoking alias effects), often exploited to explain bi-drifting within the carousel model (Ruderman \& Sutherland 1975). This includes the existing models in the literature designed to explain bi-drifting. Circulating beamlets along a single closed loop can in special cases result in bi-drifting. This has been demonstrated explicitly in the case of elliptical loops (Wright \& Weltevrede 2017, and as described elsewere in these proceedings). Sufficiently distorted circulation paths appear to be rare given that only two bi-drifting pulsars are known.

\section{PSRSalsa}

PSRSalsa (a Suite of ALgorithms for Statistical Analysis of pulsar data) has been used to obtain the results presented here. PSRSalsa includes an ever growing set of tools, including those allowing (interactive) visualisation, various types of fluctuation analysis, the quantification and fitting of single-pulse flux distributions and polarization properties. It works with standard pulsar data formats, such as PSRFITS. The software comes with a tutorial to get you started and the author is happy to be contacted for advice about its use. PSRSalsa is freely available (https://github.com/weltevrede/psrsalsa or http://www.jb.man.ac.uk/ pulsar/Resources/psrsalsa.html). If youre interested in single-pulse or polarization studies of pulsars, why not try out PSRSalsa to facilitate your research?

\section{References}

Champion, D. J., et al. 2005, MNRAS, 363, 929

Ruderman, M. A. \& Sutherland, P. G. 1975, ApJ, 196, 51

Weltevrede, P. 2016, A\& A, 590, 109

Wright, G. A. E. \& Weltevrede, P. 2017, MNRAS, 464, 2597 